\title{
MedienPädagogik
}

Zeitschrift für Theorie und Praxis der Medienbildung

\section{Die berufliche Tabletaneignung von Lehrkräften als Beispiel der Mediatisierung pädagogischer Handlungskontexte}

\section{Theoretische Perspektiven und empirische Befunde}

\author{
Lara Gerhardts, Lukas Dehmel und Dorothee M. Meister
}

\section{Zusammenfassung}

Die Digitalisierung von Schule und Unterricht weiter voranzubringen, gehört zu den erklärten Zielen der Bildungspolitik. Dementsprechend werden inzwischen immer mehr Digitalisierungsprojekte gefördert, wobei die schulische Ausstattung mit Tablets bereits seit einigen Jahren und nach wie vor einen Schwerpunkt bildet. Im Rahmen dieses Beitrags gehen wir anhand von qualitativen Analysen empirischen Datenmaterials aus unserer Begleitforschung zu einer städtischen Ausstattungsinitiative der Frage nach, wie die berufliche Aneignung von Diensttablets durch Lehrkräfte - als einem elementaren Bestandteil der tiefgreifenden Mediatisierung des schulischen Arbeitsalltags - im Einzelnen abläuft und welche Einflussfaktoren dabei eine Rolle spielen. Mit Rekurs auf die Theorie einer «Pädagogik der Dinge» (Nohl 2011) interpretieren wir den Aneignungsprozess als Ausbildung eines «HYbrid-Akteurs» (ebd., 101) aus Lehrkraft und mobilem Endgerät. Bedingt durch technische Weiterentwicklungen fordert das Tablet die Medienkompetenz der Lehrkraft im Rahmen ihres beruflichen Handelns immer wieder neu heraus, sodass sich ein fortwährender Wechsel zwischen Phasen der Hand- 
lungssicherheit und Phasen der Irritation ergibt. Wie schnell und wie gut eine Lehrkraft technikinduzierte Irritationen überwinden und sich an neue mediale Gegebenheiten anpassen kann, hängt von verschiedenen Faktoren ab. Als besonders aneignungsrelevant konnten wir basierend auf unseren empirischen Daten erstens die allgemeine Grundhaltung zum schulischen Einsatz digitaler Medien identifizieren; zweitens die bereits vorhandene Medienkompetenz der Lehrkraft bzw. ihre diesbezügliche Selbsteinschätzung; und drittens die Unterstützung, die der Lehrkraft während des Aneignungsprozesses zuteil wird. Im Sinne einer möglichst effizienten Integration des Tablets in das gesamte berufliche Handlungsspektrum von Lehrkräften lassen sich hinsichtlich aller drei genannten Einflussfaktoren gewisse Optimierungspotenziale aufzeigen.

\title{
The Acquisition of New Skills in the Use of Tablet Computers. School Teachers' Learning Practices as an Example of Mediatization in Pedagogical Contexts
}

\begin{abstract}
More and more effort is put into the digitalization of schools. In times of mobile learning, tablet computers play an important role in this context. This article aims at reconstructing the practices of school teachers as to their tablet-related skill acquisition, which is an important aspect of mediatization within their working life. We also analyze the key factors influencing these acquisition processes. Our findings are based on a qualitative analysis of empirical data gathered in different schools which have recently been equipped with teacher tablets as part of a vast digitalization project. We adopt the transactional perspective of a «pedagogy of things» (Pädagogik der Dinge, Nohl 2011) and conceptualize the relationship between the teacher and the tablet as one of reciprocal impact that finally leads to the formation of a «hybrid actor» (HYbrid-Akteur, ibid., 101). In this compound, technical innovations force the teacher to constantly try and gain new skills. Phases of temporary stability (i. e. skillful routine) and phases of irritation (i. e. lack of knowledge) consistently alternate with each other. Quality and pace of tablet-related acquisition processes depend on several determining factors. According to our analysis, there are three crucial ones: firstly, the general
\end{abstract}


attitude towards the use of digital media in school; secondly, the previous media literacy of the teacher and his or her respective self-perception; and thirdly, the support of the teacher throughout the acquisition of new work-related tablet skills. In terms of efficiently using the tablet for the whole range of a teacher's professional activities, improvements should be made regarding all the abovementioned crucial factors.

\section{Einleitung}

Bildungsinstitutionen stehen gegenwärtig vor der Herausforderung, adäquat auf die weitreichenden digitalen Transformationsprozesse innerhalb unserer Gesellschaft zu reagieren. Der Schule kommt dabei die besondere Verantwortung zu, Kinder und Jugendliche auf das Leben als mündige Bürgerinnen und Bürger in einer von tiefgreifender Mediatisierung geprägten Lebenswelt vorzubereiten (vgl. KMK 2016). Der Mediatisierungsbegriff bezeichnet einen allgegenwärtigen gesellschaftlichen Prozess, im Zuge dessen sich durch (technischen) Medienwandel - heute nun insbesondere durch die Digitalisierung - die kommunikativen Herstellungspraktiken von sozialer Wirklichkeit in sämtlichen Bereichen des öffentlichen und privaten Alltags verändern. Selbiges gilt auch vice versa: Aufgrund der engen wechselseitigen Bezüge bewirken die sich weiterentwickelnden oder gar neu entstehenden kommunikativen Praktiken ihrerseits Veränderungen innerhalb des Mediensystems, woraufhin Menschen dann wiederum beginnen, anders zu kommunizieren usw. Letztlich müssen sich Machtgefüge permanent neu ausbalancieren, was einen fortwährenden Wandel von Bedürfnissen, Inhalten und Ästhetiken bedingt (vgl. Krotz 2001, 2017).

Auch und insbesondere Lehrerinnen und Lehrer stehen in der Pflicht, sich mit Prozessen tiefgreifenden medialen und kommunikativen Wandels auseinanderzusetzen und sich immer wieder neu zu orientieren, um ihrer professionellen Verantwortung unter sich verändernden Bedingungen weiterhin gerecht werden zu können. Diesbezüglich stellt sich der Status quo an Schulen in Deutschland bislang allerdings ernüchternd dar: So verweisen die Ergebnisse empirischer Studien seit Jahren darauf, dass der unterrichtliche Einsatz digitaler Medientechnologien insgesamt noch recht zurückhaltend erfolgt und deutsche Schulen hier im internationalen 
Vergleich zurückliegen (vgl. Eickelmann et al. 2019). Was die Digitalisierung und folglich auch Prozesse (tiefgreifender) Mediatisierung betrifft, zeigen sich somit erhebliche Diskrepanzen zwischen dem schulischen und dem ausserschulischen Alltag von Kindern und Jugendlichen. Als Gründe werden häufig die schlecht gewarteten IT-Infrastrukturen und die fehlende Verbindlichkeit bei der Entwicklung und Umsetzung geeigneter Medienkonzepte für Schulen angeführt; auch unzureichende Medienkompetenzen und ein tendenziell medienskeptischer Habitus auf Seiten der Lehrkräfte haben sich als problematisch erwiesen. Zudem mangelt es offensichtlich an zeitgemässen Fortbildungskonzepten, um Lehrerinnen und Lehrern den Zugang zu digitalen Medien zu erleichtern (vgl. Eickelmann et al. 2019; Drossel et al. 2019; Kommer 2016; Petko 2012).

Um Digitalisierungshemmnissen auf Ebene der IT-Ausstattung entgegenzuwirken, werden von politischer Seite inzwischen vermehrt Finanzierungsanstösse gegeben (wie etwa durch die Ausstattungsinitiative des Bundes oder die Verabschiedung des Digitalpaktes). Im Sinne einer nachhaltigen Anregung des schulischen Medieneinsatzes auf einem tatsächlich lernförderlichen Niveau bedarf es allerdings mehr als nur der Bereitstellung finanzieller Mittel - oder zugespitzt formuliert: Die Digitalisierung von Schule allein schafft noch keine hinreichende Grundlage für einen pädagogisch 〈wertvollen〉 Mediatisierungsprozess. Zusätzlich gilt es, auch die angesprochenen pädagogisch-konzeptionellen sowie personellen $\mathrm{Me}$ diatisierungshemmnisse zu beseitigen. Die dafür zu leistende Erarbeitung geeigneter Medienkonzepte für Schulen bzw. bedarfsgerechter Aus- und Fortbildungskonzepte für Lehrerinnen und Lehrer setzt zunächst eine möglichst genaue Kenntnis jener Prozesse und kontextuellen Bedingungen voraus, die der Aneignung und Nutzung digitaler Medien durch Lehrpersonen zugrunde liegen (und die aus Perspektive der Mediatisierungstheorie wiederum auf mediale Weiterentwicklungen zurückwirken können (siehe oben)). So bedarf es umfangreicher Grundlagenforschung, um fundierte Empfehlungen für die Verankerung digitaler Medien im Schulalltag wie auch für die Aus- und Fortbildung von Lehrpersonen geben zu können.

Im Zuge der beschriebenen Ausstattungsinitiativen hat sich inzwischen ein breit angelegter Forschungsdiskurs zum schulischen Lehren und 
Lernen mit digitalen Medien herausgebildet. In aktuellen Untersuchungen spielt insbesondere der innerhalb wie ausserhalb der Wissenschaft viel diskutierte Einsatz von Tablets eine zentrale Rolle (vgl. z.B. Meister und Mindt 2020, Bastian und Aufenanger 2017 oder auch Friedrich et al. 2015). Aus Perspektive der Mediatisierungsforschung geht es dabei um den Wandel etablierter pädagogischer Kommunikationspraxen unter zunehmend digitalisierten Rahmenbedingungen. Bislang liegen diesbezüglich recht wenige, eher auf bestimmte Einzelaspekte ausgerichtete Arbeiten vor (vgl. etwa Tilmann 2018). Dabei bleibt der fokussierte Blick auf die berufsbezogene Aneignung mobiler Medientechnologien durch Lehrkräfte noch weitgehend desiderabel. Unseres Erachtens stellt gerade dieser Aspekt einen wichtigen Teilprozess der Mediatisierung von Schule dar, weshalb diese Forschungslücke im Rahmen unseres Beitrags adressiert werden soll. Unsere leitenden Forschungsfragen lauten: (a) Wie läuft die Aneignung des Tablets durch die Lehrkräfte im Zuge der Mediatisierung ihrer beruflichen Handlungskontexte im Einzelnen ab? (b) Welche förderlichen bzw. hinderlichen Einflussfaktoren lassen sich in Bezug auf diesen Aneignungsprozess identifizieren?

Ideale Bedingungen für die Erforschung medialer Aneignungsprozesse bieten Settings, in denen die Aneignung nicht durch Digitalisierungshemmnisse auf Ebene der IT-Ausstattung (siehe oben) konterkariert wird. Ein in diesem Sinne idealtypisches Forschungsfeld stellt die Ausstattungsinitiative einer nordrhein-westfälischen Grossstadt dar, die durch den Arbeitsbereich Medienpädagogik und empirische Medienforschung der Universität Paderborn wissenschaftlich begleitet wird. Die Leitidee dieses Programms ist der Aufbau einer lernförderlichen, schulübergreifend einheitlichen und somit wartungsarmen IT-Infrastruktur an allen Schulen der Stadt. Die Grundausstattung beinhaltet flächendeckendes WLAN, digitale Präsentationstechnik in sämtlichen Unterrichtsräumen, einen speziell eingerichteten Cloudspeicher sowie persönliche E-Mailadressen für alle Nutzerinnen und Nutzer. Darüber hinaus erhalten derzeit alle Lehrkräfte der städtischen Schulen ein eigenes Dienst-iPad, dessen Aneignung wir im Folgenden in den Mittelpunkt unserer Analyse stellen. Bereits abgeschlossen ist die flächendeckende Ausgabe der Geräte im Primarbereich, weshalb 
unser Begleitforschungsprojekt an ausgewählten städtischen Grundschulen stattgefunden hat. ${ }^{1}$

Im nächsten Abschnitt (Punkt 2) beziehen wir uns zur theoretischen Fundierung der anschliessenden Analysen zunächst auf Nohls Arbeiten zu einer «Pädagogik der Dinge», um das für uns leitende Konzept des Verhältnisses zwischen Mensch und Medientechnologie näher zu bestimmen. Mit Nohl (2011) greifen wir auf eine explizit erziehungswissenschaftliche Reflexion und Deutung zentraler Konzepte der Akteur-Netzwerk-Theorie (ANT) zurück. Die seitens der Mediatisierungsforschung berechtigterweise aufgezeigten Limitationen der ANT für medien- und sozialwissenschaftliche Untersuchungen (vgl. z. B. Couldry 2008) spielen angesichts des hier verfolgten Forschungsanliegens eine eher randständige Rolle und können daher im Weiteren vernachlässigt werden. Auf Basis unserer theoretischen Annahmen zum Verhältnis zwischen menschlichem Akteur (der Lehrperson) und dinglichem Aktanten (dem Tablet) entwickeln wir im anschliessenden Abschnitt das forschungsmethodische Vorgehen unseres Begleitforschungsprojekts (Punkt 3). Es folgt eine Darstellung der ersten empirischen Erkenntnisse. Unsere Befunde bezüglich der Tabletaneignung von Lehrerinnen und Lehrern überführen wir in ein an Nohl angelehntes Prozessmodell. Des Weiteren arbeiten wir relevante Einflussfaktoren heraus, denen innerhalb des Aneignungsprozesses eine präfigurierende Wirkung zukommt (Punkt 4). Unser Beitrag schliesst mit ersten Implikationen für gezielte Optimierungsmassnahmen, die angesichts unserer Forschungsergebnisse geeignet erscheinen, Lehrerinnen und Lehrern die professionelle Nutzung von Diensttablets zu erleichtern (Punkt 5).

1 Es sei ausdrücklich darauf hingewiesen, dass dieser Beitrag keine rein grundschulpädagogische Perspektive einnimmt. Die Entscheidung, den Feldzugang auf den Grundschulbereich zu beschränken, ist rein forschungspragmatisch begründet: Während unseres Untersuchungszeitraums war die Ausgabe der Diensttablets an allen anderen Schulformen noch nicht abgeschlossen, weshalb keine für unsere Zwecke ergiebigen Daten zu erwarten gewesen wären. Grundsätzlich lässt sich bereits an dieser Stelle vorwegnehmen, dass unsere in den Grundschulen gewonnenen Erkenntnisse sich als wenig schulformspezifisch erwiesen haben. Wie die weiteren Ausführungen zeigen werden, sind einzelne Aspekte des Nutzungsverhaltens der Lehrpersonen natürlich durch den Grundschulkontext bedingt (z. B. die Verwendung bestimmter Apps im Unterricht). Im Grossen und Ganzen ist aber anzunehmen, dass sich die herausgearbeiteten Aneignungsmuster und Einflussfaktoren (vgl. Punkt 4) ähnlich auch bei Lehrkräften anderer Schulformen finden lassen. 


\section{2. «Tiefgreifende Mediatisierung» und «Pädagogik der Dinge» als theoretische Bezugspunkte}

Das Mediatisierungskonzept geht von einer durch den Medienwandel permanent angetriebenen Umwälzung der kommunikativen Herstellungspraktiken sozialer Wirklichkeit aus (vgl. Krotz 2001). Sowohl für die kommunikative Konstruktion heutiger Gesellschaftsentwürfe auf einer Makroebene als auch für die Soziogenese kleinerer sozialer Zusammenhänge auf einer Meso- oder Mikoebene stellen digitale Kommunikationsmedien mittlerweile eine konstitutive Voraussetzung dar, weshalb diesbezüglich inzwischen von einer "tiefgreifenden Mediatisierung» (deep mediatization) gesprochen wird (vgl. Hepp 2016). Als Charakteristika zeigen Hepp und Hasebrink fünf unterschiedliche Merkmale auf, die den Alltag der Menschen bis ins letzte Detail durchdrungen haben und kulturellen und gesellschaftlichen Wandel im Grossen wie im Kleinen bedingen bzw. massgeblich vorantreiben: 1. die Differenzierung technischer Kommunikationsmedien; 2. eine durch diese Kommunikationsmedien permanent ermöglichte Konnektivität; 3. die Allgegenwärtigkeit von Medien in sämtlichen Lebensbereichen, 4. eine hohe und beschleunigte technologische Innovationsdichte; und 5. eine zunehmende Datafizierung des gesellschaftlichen Zusammenlebens (vgl. Hepp und Hasebrink 2017, 335ff.; Hepp und Hasebrink 2018, 19ff.).

Grundlegend für diesen Beitrag ist die Annahme, dass es zu kurz greifen würde, das Tablet im Zuge tiefgreifender Mediatisierungsprozesse innerhalb der Schule als reines «Werkzeug» der Lehrkraft zu deuten. Eine solche Sichtweise würde das transformative Eigenpotenzial digitaler Medien missachten, das gerade in pädagogischen Handlungskontexten bedeutsam ist (vgl. hierzu auch Allert und Asmussen 2017). Zur Überwindung einer rein instrumentellen und damit notwendig verkürzenden Perspektive auf das Tablet in unterrichtlichen Zusammenhängen schlägt Welling als theoretischen Analyserahmen Nohls Ausarbeitungen zu einer «Pädagogik der Dinge» vor (vgl. Welling 2017), die wir nachfolgend auch unserer Forschung zugrunde legen.

Vor dem Hintergrund der benannten theoretischen Bezugspunkte verstehen wir die Synthese aus Lehrperson und Tablet in schulbezogenen Handlungskontexten als einen «Hybrid-Akteur» aus Mensch und materiellem 〈Ding (Nohl 2011, 101) und interpretieren die Aneignung und die sich 
etablierende Nutzung des Endgeräts als einen reziproken Austauschprozess, in dem der menschliche Akteur und der dingliche Aktant gegenseitig aufeinander einwirken. Durch die wechselseitigen Bezüge zwischen Akteur und Aktant wird der Hybrid-Akteur überhaupt erst hervorgebracht und dann in seiner Weiterentwicklung permanent angetrieben (vgl. Nohl 2011, 92; Nohl und Wulf 2013, 5ff.). Gemäss der «Pädagogik der Dinge» lassen sich die Wechselwirkungen zwischen dem technischen und dem menschlichen Part wie folgt fassen:

Das Tablet gibt durch seine materiellen und medialen Eigenschaften einen Möglichkeitsraum vor, der potenzielle Verwendungsweisen präfiguriert. Dieser technikseitig bedingte Möglichkeitsraum legt bestimmte Praktiken im Umgang mit dem Tablet nahe, wohingegen andere Praktiken weniger naheliegend sind oder gar ausgeschlossen werden (vgl. Nohl 2011, 98). Zu betonen ist, dass die Funktionalität eines Tablets als digitalem 〈Ding〉 nicht mehr so stark an den Aspekt der Materialität gekoppelt ist wie bei analogen Dingen der Fall. Der Terminus des Digitalen beschreibt «das binäre elektronische (digitale) System [...], welches in heutigen Computern und anderen elektronischen Geräten verwendet wird» (Hartmann und Wimmer 2011, 9f.). Dieses ist die Grundlange für eine «konvergente Verbundnutzung verschiedener Präsentationsformen wie Bild, Text, Grafik, Animation, Film, Sprache und Musik» (Hüther 2005, 347) über ein einzelnes Endgerät. Hinsichtlich der möglichen Verwendungsweisen des Tablets sind diese medial hergestellten Präsentationsformen bzw. Nutzungsmöglichkeiten sehr viel entscheidender als die Materialität des Gerätes im engeren Sinne. Um diesem Umstand Rechnung zu tragen, sprechen wir im weiteren Verlauf unseres Beitrags von der «medial-materiellen» Beschaffenheit des Möglichkeitsraumes (Dehmel und Zick 2020, 183). Die Aktivität der Lehrperson als menschlichem Akteur besteht darin, den durch die Technik eröffneten und zugleich auch begrenzten Spielraum mittels seiner Fähigkeit zu Reflexion und divergentem Denken (Nohl 2011, 98) kreativ und zielgerichtet zu nutzen. So lassen sich diverse Nutzungsideen für eine Vielzahl von beruflichen Anwendungskontexten entwickeln. Dabei kommt es im Laufe solcher Austauschprozesse zwischen dem menschlichen und dem technischen Part eines Hybrid-Akteurs laut Nohls Theorie immer wieder zur soziodinglichen Genese neuer «Dingfunktionen». Nohl 
beschreibt damit die Entdeckung von bislang unbekannten Verwendungsvarianten von Gegenständen, die deutlich von jenen «Handlungsprogrammen» abweichen können, die dem Gegenstand eingeschrieben (d. h. seitens des Herstellers intendiert) sind (vgl. ebd., 96ff.). In Reaktion darauf, dass der Akteur den Möglichkeitsraum andersartig nutzt als ursprünglich gedacht, kommt es dann unter Umständen zur Weiterentwicklung entsprechender Technologien, um den Aktanten den erkennbar gewordenen Bedarfen der Zielgruppe anzupassen. Dadurch entsteht wiederum ein neuer Möglichkeitsraum usw.

Dieses interdependente Mensch-Technik-Verhältnis im Sinne der Formierung von Hybrid-Akteuren entwirft Nohl unter Rückgriff auf Latours Überlegungen zur Akteur-Netzwerk-Theorie (ANT) (vgl. Latour 1998), die er allerdings pädagogisch perspektiviert und entscheidend weiterentwickelt. So hebt Nohl (mit Rekurs auf Rammert und Schulz-Schaeffer 2002) deutlich hervor, dass (bislang) nur der Mensch über jene Art von (Selbst-) Reflexionsfähigkeit verfügt, die es in kritischen Situationen ermöglicht, auch verfestigte soziodingliche Handlungsroutinen zu überdenken und ggf. bewusst aufzubrechen (vgl. Nohl 2011, 43ff.; Nohl 2013, 192f.). Zwar übernimmt Nohl das aus der ANT stammende Konzept eines Austauschverhältnisses zwischen Mensch und Technik; allerdings dahingehend relativiert, dass der menschliche Akteur als derjenige Part angenommen wird, der die gemeinsamen Handlungen des Hybrid-Akteurs insgesamt reflektiert und mittels seiner Fähigkeit zum intentionalen Handeln auch zu steuern vermag (vgl. ebd.).

Die Ausbildung eines handlungsfähigen Hybrid-Akteurs konzeptualisiert Nohl im Anschluss an Peirce (und dessen Rezeption durch Dewey (1989)) in drei aufeinanderfolgenden Phasen, die wir im Folgenden zur Grundlage unserer Überlegungen machen:

- einer ersten Phase (der «Erstheit»), die die Ausbildung «spontaner Gefühlsqualitäten» beinhaltet, wenn Mensch und Ding zum ersten Mal aufeinandertreffen (bei uns als Phase der affektiven Erstreaktionen bezeichnet);

- einer zweiten Phase (der «Zweitheit»), in der auf Basis der ersten «Gefühlsqualitäten» durch initiale Austauschprozesse zwischen Mensch 
und Ding verschiedene Umgangsformen entwickelt und erprobt werden (in unserer Untersuchung die Phase der tentativen Austauschpraktiken);

- und einer dritten Phase (der «Drittheit»), in der sich die herausgebildeten Austauschpraktiken zwischen Mensch und Ding stabilisieren und in Alltagsroutinen übergehen (bei uns die Phase der routinierten Austauschpraktiken) (vgl. Nohl 2011, 101f.)

Nohl verweist ausdrücklich darauf, dass sich der am Ende dieser drei Phasen ausgebildete Hybrid-Akteur aus Mensch und Ding angesichts technischer Innovationen, gesellschaftlicher Entwicklungen usw. auch danach permanent weiterentwickelt und sowohl den Menschen als auch den (technischen) Gegenstand fortlaufend verändert (vgl. ebd.). Die Verbindung von Mensch und Ding ist folglich nie bzw. immer nur temporär 〈fertig〉 - wie unsere Analysen zeigen werden, gilt dies unter Beteiligung medial-materieller Dinge wie dem Tablet in ganz besonderen Masse (siehe unten). In fortgeschrittenen Entwicklungsstadien des Hybrid-Akteurs, so unsere These, entwickeln sich gleichwohl zunehmend Routinen im Umgang mit 〈Ding〉induzierten Irritationen und Ungewissheiten, die zu deren schneller und lösungsorientierter Überwindung beitragen und dadurch mit einer gewissen grundlegenden Stabilisierung der Austauschpraktiken einhergehen.

\section{Forschungsmethodik}

Die im Anschluss an Nohl konzipierte 3-Phasen-Struktur der Herausbildung von Hybrid-Akteuren (siehe Punkt 2) nutzen wir im Rahmen unserer empirischen Untersuchung als Forschungsheuristik:

Die Datenerhebung wurde folglich gezielt so angelegt, dass ergiebiges Datenmaterial im Hinblick auf affektive Erstreaktionen, tentative und (zunehmend) routinierte Austauschpraktiken anzunehmen war. Dafür wurden mehrere Erhebungsmethoden trianguliert, wobei leitfadengestützte qualitative Interviews und Gruppendiskussionen mit Lehrkräften im Mittelpunkt unserer Erhebungen standen und zusätzlich Unterrichtsbeobachtungen im Sinne einer Anreicherung unseres Datenmaterials durchgeführt wurden. Die Datenerhebung fand an drei verschiedenen städtischen 
Grundschulen statt. ${ }^{2}$ Innerhalb dieses Rahmens wurde im Sinne maximaler Kontrastierung ein möglichst heterogenes Sample angestrebt: An allen Erhebungsschulen konnten Daten unter Beteiligung von Lehrerinnen und Lehrern in ganz unterschiedlichen Funktionen generiert werden, angefangen bei Fach- und Klassenlehrerinnen und -lehrern über medienbeauftragte Lehrkräften und Sonderpädagoginnen und -pädagogen bis hin zu den Schulleitungen. Der erste Erhebungszeitraum fand sechs Monate nach Aushändigung der Diensttablets statt. Nach Abschluss des ersten Erhebungszeitraums bestand unsere auswertbare Datenbasis aus acht qualitativen Interviews, drei Gruppendiskussionen und 13 Unterrichtsbeobachtungen in verschiedenen Klassenstufen. Die Datenauswertung erfolgte nach den Regeln der strukturierenden qualitativen Inhaltsanalyse (vgl. Kuckartz 2016). Im Sinne einer deduktiv-induktiven Form der Kategorienbildung wurden die unter Punkt 2 beschriebenen theoretischen Konzepte als Ausgangskategorien herangezogen und empirisch weiter ausdifferenziert. Um ein längsschnittliches Forschungsdesign umzusetzen, ist vorgesehen, im Abstand von einem Jahr (d. h. 18 Monate nach Aushändigung der Diensttablets) einen zweiten Erhebungszeitraum anzuschliessen. Nach Möglichkeit sollen dann im Austausch mit denselben Probandinnen und Probanden weitere Daten erfasst werden, die über die Momentaufnahmen hinaus auch Entwicklungstendenzen zugänglich machen und eine entsprechende Verlaufsanalyse ermöglichen.

\section{Ausgewählte Ergebnisse}

Unsere bisherigen Analysen basieren auf den während des ersten Erhebungszeitraums (sechs Monate nach Aushändigung der Tablets) generierten Daten. Sie geben somit Aufschluss über den Verlauf tabletbezogener Aneignungsprozesse in einem anfänglichen Stadium, d. h. also vor allem über die erste Ebene der Ausbildung des Hybridakteurs (siehe Abb. 1:

2 Zur Begründung des auf die Grundschule beschränkten Feldzugangs siehe Fussnote 1. 
Phasen der 1. Ebene). ${ }^{3}$ Bezogen auf das Anfangsstadium der Tabletaneignung lassen sich bereits einige interessante Analyseergebnisse berichten:

Erstens untermauert unsere detailgenaue qualitative Datenauswertung die Passung zwischen der eingangs theoriegeleitet entworfenen Phasenstruktur des Aneignungsprozesses (siehe Punkt 2) und dem empirischen Material. Die Befunde zur Grundstruktur des Aneignungsprozesses bündeln wir unter Punkt 4.1.

Zweitens liefert die Datenauswertung differenzierte Erkenntnisse bezüglich der verschiedenen Einflussfaktoren, von denen abhängt, wie erfolgreich der Aneignungsprozess verläuft - in seinem Anfangsstadium und womöglich auch darüber hinaus. ${ }^{4}$ Unter Punkt 4.2 stellen wir jene Einflussfaktoren vor, die sich bereits auf Basis der Daten des ersten Erhebungszeitraums als besonders aneignungsrelevant identifizieren lassen.

\subsection{Ablauf des Aneignungsprozesses}

Anders als es vielleicht bei weniger komplexen (nicht-digitalen) Dingen der Fall sein mag, kann man beim Tablet - als komplexem medial-materiellen Artefakt - nicht von der Aneignung sprechen. So beschränken sich tabletbezogene Aneignungsprozesse nicht allein auf die grundlegende Handhabung des Geräts, sondern finden darüber hinaus immer auch bereichsspezifisch in Bezug auf die jeweils genutzte Software (z.B. spezifische Apps) statt. Im Umgang damit bildet sich ein immer vielschichtiger werdender Hybrid-Akteur mit einer Reihe unterschiedlicher Dingfunktionen heraus. In unseren empirischen Daten reichen diese Funktionen von der Darstellung von Arbeitsaufgaben, Unterrichtsinhalten (insbesondere mittels der App «Explain Everything») und Arbeitsergebnissen der Schülerinnen und

3 Sehr vereinzelt zeichnen sich in den Daten des ersten Erhebungszeitraums auch schon Ansätze der Weiterentwicklung des Hybrid-Akteurs ab (siehe Abb.1: Phase der tentativen Austauschpraktiken auf der 2. Ebene), wie etwa in dem nachfolgend unter Punkt 4.1 beschriebenen Anschauungsbeispiel. Der geplante zweite Erhebungszeitraum (18 Monate nach Aushändigung der Tablets) dürfte in Bezug auf die Weiterentwicklung des Hybrid-Akteurs dann aber deutlich umfangreicheres und aussagekräftigeres Datenmaterial erbringen.

4 Inwieweit die identifizierten Einflussfaktoren den Verlauf des Aneignungsprozesses auch über das Anfangsstadium hinaus beeinflussen, wird erst eine vergleichende Analyse der Daten unseres zweiten Erhebungszeitraums zeigen. 
Schüler sowie dem Einsatz fachspezifischer Übungsprogramme (in unserem Sample insbesondere die Mathe-App «Blitzrechnen», die Deutsch-App «Antolin» oder die fachübergreifende App «Anton») über die Umsetzung fächerverbindender Foto- und Videoprojekte bis hin zur Klassenorganisation. Die von Nohl thematisierte Veränderung des dinglichen Aktanten wird im Falle digitaler Geräte besonders virulent, da sich das Tablet bzw. der durch das Tablet eröffnete medial-materielle Möglichkeitsraum innerhalb des Hybrid-Akteurs im Zuge von Updates des Betriebssystems, durch Softwareupdates oder gar durch die Installation gänzlich neuer Apps jedes Mal mehr oder weniger gravierend verändert und weiter ausdifferenziert. Hier schlägt sich das von Hepp und Hasebrink (2017) aufgezeigte Charakteristikum einer hohen und beschleunigten technologischen Innovationsdichte im Zuge einer tiefgreifenden Mediatisierung (siehe Punkt 2) nieder. Für weniger technikaffine Lehrkräfte kann dies sehr heraus- oder sogar überfordernd sein.

«Wir hatten auch eine Kollegin, die hatte echt riesiges Pech, [...] hat ihr iPad eingerichtet. Ähm ist über Nacht dann ähm gab es ein Update und am nächsten Morgen war alles weg. [...] Ja, das war PECH, ne? Und ähm von daher, da stossen schon einige an ihre Grenzen, ich auch manchmal.» (Frau D.; Grundschullehrerin/Medienbeauftragte im Einzelinterview)

Die Lehrpersonen sehen sich aufgrund der tiefgreifenden Mediatisierung ihres beruflichen Alltags folglich immer wieder mit neuen technikseitig evozierten Herausforderungen konfrontiert, die sie zur Veränderung bzw. Anpassung ihrer Denk- und Handlungsschemata in Bezug auf den Einsatz des Tablets veranlassen. So kommt es zu einem permanenten Changieren zwischen einem Zustand der (temporären) Sicherheit und plötzlicher Irritation, die immer wieder eine erneute Auseinandersetzung mit dem Tablet und entsprechende Modifikationen im Umgang damit erfordert.

Zur Veranschaulichung geben wir ein Beispiel aus unseren Unterrichtsbeobachtungen: Eine eher wenig technikaffine Lehrerin unseres Samples hatte sich in mühsamer Auseinandersetzung mit dem Tablet also durch das Durchlaufen der drei Phasen der affektiven Erstreaktionen, 
der (ersten) tentativen Austauschpraktiken und der (ersten) routinierten Austauschpraktiken (siehe Abb.l, 1. Ebene) - angeeignet, wie sich mithilfe der App «Explain Everything» handgefertigte Zeichnungen erstellen, durch die Kopplung mit dem Whiteboard für alle im Raum Anwesenden sichtbar machen und mithilfe der Radiergummi-Funktion beliebig oft verändern lassen. Die dafür notwendigen Schritte beherrschte sie nach mehrmaligem Üben sicher, sodass sich der Austausch mit dem Tablet bezüglich dieser speziellen Dingfunktion innerhalb der täglichen Handlungsroutinen zunächst stabilisiert hatte (Zustand der temporären Sicherheit). Durch ein Softwareupdate veränderte sich dann allerdings das Symbol für die Radiergummi-Funktion innerhalb der App. Während unserer Unterrichtsbeobachtung kam es zu einer Situation, in der die Lehrerin kurzfristig verunsichert war, weil sie die gewünschte Funktion auf dem Tabletinterface nicht mehr direkt identifizieren konnte (plötzliche Irritation). Durch die Veränderung des Tablets bzw. von dessen medial-materiellem Möglichkeitsraum war die Lehrerin nun technikseitig aufgefordert, sich erneut mit dem Gerät auseinanderzusetzen. Im Zuge dieser Auseinandersetzung entdeckte sie schliesslich das neue äquivalente Symbol und konnte die Irritation somit selbstständig überwinden.

In diesem einfachen Beispiel zeigt sich, wie die Veränderung des Tablets (als technischem Aktanten) unmittelbar zu Veränderungen auf Seiten der Lehrkraft (als menschlichem Akteur) (auffordert). Nun liegt es an dem jeweiligen Akteur, dieser Handlungsaufforderung nachzukommen oder aber nicht nachzukommen. Sofern der Akteur entscheidet, sein Handeln an den neuen Stand der Technik anzupassen und dabei auf Kompetenzen aufbaut, die er im Zuge seines bisherigen Medienhandelns bereits erworben hat, entsteht im Idealfall eine spiralförmige Dynamik, die mit fortschreitendem Aneignungsprozess auf immer höhere Ebenen des Austauschs zwischen Akteur und Aktant und somit zur Weiterentwicklung des Hybrid-Akteurs insgesamt führt. Wir haben die Ausbildung und permanente Weiterentwicklung des Hybrid-Akteurs aus Lehrkraft und Tablet auf Basis unserer empirischen Befunde in eine an Nohl angelehnte Heuristik überführt, die die Grundstruktur des Aneignungsprozesses modelliert (siehe Abb. 1). 


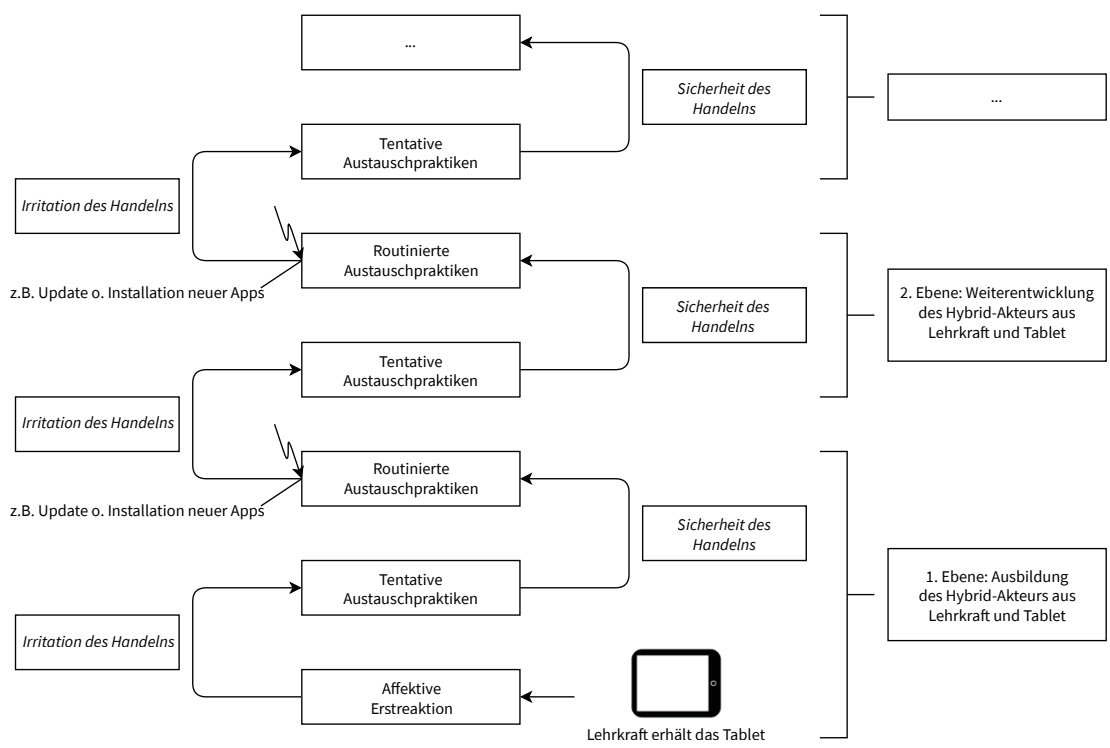

Abb. 1.: Ausbildung und Weiterentwicklung eines Hybrid-Akteurs aus Lehrkraft und Tablet in Anlehnung an Nohl 2011 (eigene Darstellung).

Das erste Durchlaufen der drei Phasen der affektiven Erstreaktionen, der tentativen Austauschpraktiken und der routinierten Austauschpraktiken bildet das Fundament für die Ausbildung neuer Dingfunktionen innerhalb der von Mediatisierungsprozessen durchzogenen beruflichen Handlungskontexte von Lehrkräften und stellt somit die initiale Voraussetzung für eine erfolgreiche weitere Auseinandersetzung mit spezifischeren unterrichtlichen Einsatzmöglichkeiten des Gerätes dar (d. h. für erneute Durchläufe von Phasen tentativer und schliesslich routinierter Austauschpraktiken auf zunehmend höheren Kompetenzebenen).

\subsection{Den Aneignungsprozess beeinflussende Faktoren}

Anhand unseres Datenmaterials lässt sich des Weiteren erkennen, dass das Qualitätsniveau wie auch das Tempo tabletbezogener Aneignungsprozesse von Lehrkräften verschiedenen Einflussfaktoren unterliegen. Drei übergeordnete Faktoren erweisen sich unseren Analysen zufolge als besonders aneignungsrelevant: die Grundhaltung zu digitalen Medien im Schulkontext, die Medienkompetenz und diesbezügliche Selbsteinschätzung der Lehrkräfte sowie deren Unterstützung im Verlauf der Aneignung. 


\subsubsection{Grundhaltung zu digitalen Medien im Schulkontext}

Ein erster wichtiger Faktor, der sich je nach Ausprägung entweder förderlich oder auch hemmend auf die Tabletaneignung von Lehrkräften auswirken kann, betrifft die grundlegende Haltung gegenüber dem schulischen Einsatz digitaler Medien. Als prägend erweist sich in diesem Zusammenhang zunächst, welche Leitvorstellungen diesbezüglich seitens der Schulleitung propagiert werden. Die institutionell vorherrschende medienbezogene Grundhaltung wirkt sich teils unmittelbar, teils auch vermittelt über die vor diesem Hintergrund gewachsenen persönlichen Einstellungen der Lehrkräfte auf den Verlauf des Aneignungsprozesses aus.

Was die institutionelle Grundhaltung betrifft, zeigt sich in den Daten, dass die Schulleitungen unseres Samples der Implementation von Tablets nicht nur positiv gegenüberstehen, sondern diese sogar nachdrücklich von den Lehrkräften einfordern.

«Und jetzt haben wir diese technischen Möglichkeiten und das heisst, die muss man natürlich auch AUSSCHÖPFEN. Das heisst, das ist jetzt auf jeden Fall ein Arbeitsschwerpunkt von uns, wie wir alle da noch fitter werden.» (Frau K.; Schulleiterin im Einzelinterview)

Die persönlichen Einstellungen der Lehrkräfte werden zumindest teilweise von dieser institutionellen Erwartungshaltung geprägt. So überrascht es wenig, dass insbesondere die affektiven Erstreaktionen der Lehrkräfte unmittelbar nach Erhalt des neuen Arbeitsgeräts (siehe Abb. 1, 1. Ebene, 1. Phase) recht positiv ausfallen, wobei die Äusserungen unserer Interviewpartnerinnen und -partner sich in einem Spektrum von kritischer Aufgeschlossenheit bis hin zu freudig-erwartungsvoller Aufgeschlossenheit bewegen. Gänzlich ablehnende Reaktionen von Lehrkräften sind innerhalb unseres Materials nicht zu finden. Insgesamt lässt sich mit Blick auf unser Sample festhalten, dass die positive Grundhaltung gegenüber digitalen Medien die Bereitschaft der Lehrkräfte, sich auf umfangreiche tentative Austauschpraktiken mit dem neuen Arbeitsgerät einzulassen (siehe Abb. 1, 1. Ebene, 2. Phase), erheblich begünstigt. 


\subsubsection{Medienkompetenz und diesbezügliche Selbsteinschätzung}

Der nächste für den Aneignungsprozess relevante Faktor ist die medienbezogene Vorerfahrung, die die einzelne Lehrkraft bei Erhalt des Tablets bereits mitbringt. Das Ausmass an vorhandener bzw. fehlender Medienkompetenz bestimmt zum einen, wo die Aneignungsspirale ihren Ausgang nimmt (d. h. auf welcher (Grundhöhe) die 1. Ebene der Ausbildung des Hybrid-Akteurs angesiedelt ist, siehe Abb. 1); und zum anderen, wie schnell die jeweils nächsten Entwicklungsebenen erreicht werden (also die 2. Ebene, 3. Ebene, usw. im Zuge der Weiterentwicklung des Hybrid-Akteurs, siehe Abb. 1). Demnach kann sowohl das Ausgangsniveau als auch die Weiterentwicklung von Hybrid-Akteuren aus Lehrkraft und Tablet stark variieren.

«Ich glaube [...] wir nehmen glaube ich einen Bruchteil überhaupt erstmal wahr. Ähm hängt dann auch ein bisschen damit zusammen wie affin in den digitalen Medien sind die Kolleginnen und Kollegen, das schwankt dann von der Nutzung sehr stark. Manche machen es ganz viel schon, manche eher weniger, weil sie sich nicht so auskennen.» (Herr H.; Grundschullehrer/Medienbeauftragter im Einzelinterview)

Die anhand der Daten unseres Samples rekonstruierbaren individuellen Kompetenzstände der Lehrkräfte sind sehr heterogen und reichen von völliger Medienfremdheit bis hin zu einer weitreichenden Medienaffinität, wobei letztere innerhalb der Kollegien aber immer noch eine Ausnahme darstellt. Dementsprechend vollziehen sich die von uns analysierten Aneignungsprozesse mehrheitlich auf basalem Niveau und in einem recht geringen Tempo.

Ein weiterer, unmittelbar mit der Medienkompetenz zusammenhängender Aspekt ist die diesbezügliche Selbsteinschätzung bzw. das daraus resultierende Selbstvertrauen der einzelnen Lehrkraft. Auch hier zeigt sich innerhalb unseres Samples eine beträchtliche Varianz, wobei sich allerdings niemand - auch nicht die befragten Medienbeauftragten der Schulen - den Status einer Expertin oder eines Experten zuschreibt. Interessanterweise werden im Zusammenhang mit der Einschätzung der eigenen medienbezogenen Kompetenzen in unseren Interviews häufig auch 
Begründungen geäussert, warum man sich gut oder eben weniger gut auskennt: Auffällig ist, dass in diesem Zusammenhang immer wieder auf das eigene Alter bzw. die eigene Generationszugehörigkeit verwiesen wird:

«Ich bin ja nun jetzt aus der jüngeren Generation und habe selbst auch zu Hause eins [ein Tablet]. Von daher wusste ich auch, okay, alles klar, da muss man ähm sich jetzt ein bisschen mehr dahinterklemmen und ich habe mir das von Anfang an zugetraut.» (Frau B.; Grundschullehrerin im Einzelinterview)

«Also ähm ich bin ja schon ne ganz andere Generation praktisch (lacht). Ähm, mich überfordert das Ganze.» (Frau P.; Grundschullehrerin in Gruppendiskussion)

Die Nutzung der Kategorie Alter bzw. Generationszugehörigkeit als eine Art 〈Legitimationsfolie〉 für mehr oder minder ausgeprägte Medienkompetenz ist in der medienpädagogischen Forschung bereits mehrfach beschrieben und diskutiert worden. Einschlägige Arbeiten gelangen zu dem Schluss, dass die simple dichotome Einteilung in eine vermeintlich medienkompetente junge Generation (die Digital Natives) und eine vermeintlich medienferne ältere Generation (die Digital Migrants) empirisch nicht haltbar ist (vgl. Burgfeld-Meise und Meister 2020; Klein 2017). So gibt es durchaus auch gegenteilige Befunde, die nahelegen, dass es gerade die älteren Lehrkräfte mit einem reichen Erfahrungsbestand sind, die sich im Falle von Irritationen und Handlungsunsicherheiten schneller auf die neuen Umstände einzustellen vermögen (vgl. Tillmann 2018, 32). Gleichwohl kann das besagte dichotome Denkschema und die (Selbst-)Einteilung in das Lager der Digital Natives bzw. der Digital Migrants das Selbstvertrauen der Beteiligten unterschiedlich beeinflussen und die Auseinandersetzung mit dem Tablet im günstigen Fall fördern, im ungünstigen Fall aber ebenso behindern oder sogar gänzlich blockieren. Tatsächlich deuten die ersten Ergebnisse unserer Analysen darauf hin, dass die Entwicklung des Hybrid-Akteurs aus Lehrkraft und Tablet letztlich primär durch begrenzte Kompetenzen und/oder eine ungünstige Selbsteinschätzung des menschlichen Akteurs limitiert wird und weit weniger durch die Grenzen des technikseitigen Möglichkeitsraumes: Letzterer bietet sehr viel Potenzial 
für den sinnvollen Einsatz von Tablets im Schulkontext. Ob allerdings potenziell nützliche Apps auch zum Einsatz gelangen oder nicht, ob verfügbare Updates tatsächlich installiert werden oder nicht usw., hängt stets davon ab, inwieweit die einzelne Lehrkraft mit der jeweiligen technischen Neuerung etwas anzufangen weiss und/oder inwieweit sie sich eine arbeitseffiziente Nutzung zutraut.

\subsubsection{Unterstützung im Verlauf der Aneignung}

Ein weiterer besonders aneignungsrelevanter Faktor ist unseren Analysen zufolge die Unterstützung, auf die Lehrkräfte während der Aneignungsprozesse zurückgreifen können. Qualität und Geschwindigkeit der Tabletaneignung hängen in hohem Masse davon ab, ob die Lehrkraft während der tentativen Phasen (siehe Abb.1, Tentative Austauschpraktiken auf 1., 2., ... usw. Ebene) eine bedarfsgerechte, an die jeweilige Entwicklungsebene angepasste Begleitung im Umgang mit dem Tablet erfährt. Nur so wird das Erreichen der nächsten temporär routinierten Phase (siehe Abb.1, Routinierte Austauschpraktiken auf 1., 2., ...usw. Ebene) gezielt unterstützt.

Aus der Forschungsliteratur zum beruflichen Lernen in der digitalisierten Arbeitswelt ist bekannt, dass wichtige Kompetenzen grösstenteils im Rahmen informeller und non-formaler Lernprozesse erworben werden. Erfolgsentscheidend ist jedoch oftmals, dass das informelle und non-formale Lernen systematisch von formalen Bildungsangeboten flankiert wird, um den angestrebten Kompetenzerwerb abzusichern (vgl. Ifenthaler 2018). Dementsprechend nehmen wir auch für tabletbezogene Aneignungsprozesse von Lehrkräften an, dass optimalerweise verschiedene informelle, non-formale und formale Lernelemente bzw. entsprechende Elemente der Lernunterstützung ineinandergreifen und sich wechselseitig ergänzen sollten. Unsere Analysen auf Basis der Daten des ersten Erhebungszeitraums geben Aufschluss darüber, welche Arten des Lernens bzw. der Lernunterstützung in den ersten sechs Monaten nach Aushändigung der Tablets zusammenspielen. Insgesamt ergibt sich das folgende Bild:

Der erste Zugang zum Gerät geschieht innerhalb eines einmaligen verpflichtenden Workshops. Innerhalb dieses strukturgebenden, wenn auch nur sehr kurzfristig bestehenden formalen Rahmens bekommen 
die Lehrkräfte ihr Tablet ausgehändigt und erhalten unmittelbar im Anschluss eine erste Einführung, in der einige grundlegende Tablet-Funktionen demonstriert werden. Bereits im Rahmen dieses Einführungsworkshops haben die Lehrkräfte Gelegenheit, verschiedene Anwendungen selbst zu erproben, womit die erste Phase tentativer Austauschpraktiken mit dem Gerät (siehe Abb.1, 1. Ebene) eingeläutet wird. Nach dem Workshop setzt sich diese erste tentative Phase weiter fort, nun aber in Form unangeleiteten Ausprobierens, was von mehreren unserer Untersuchungsteilnehmenden als «Trial and Error» charakterisiert wird. Diese tentativen Austauschpraktiken jenseits eines formalen Rahmens bilden unseren Analyseergebnissen zufolge derzeit den Kern des Aneignungsprozesses zwischen Lehrkraft und Tablet.

«Also wir hatten jetzt einmal so eine Einführung für alle. [...] Das ist erstmal viel in dem Moment. Da muss man einfach probieren, dass man da, da bisschen besser reinkommt.» (Frau N.; Grundschullehrerin/ Medienbeauftragte im Einzelinterview)

Durch seine medial-materielle Beschaffenheit stellt das Tablet diverse Anforderungen an die Lehrkraft, die es im Zuge des Aneignungsprozesses zu bewältigen gilt, d. h. die Technik gibt vor, was gelernt werden muss, um bestimmte Funktionen nutzen und somit die jeweils gewünschten Szenarien im schulischen Berufsalltag mediengestützt realisieren zu können. ${ }^{5}$

«Was ist, wenn ich jetzt ein Bild suche über Google? Wie finde ich das überhaupt? Wie speichere ich das ab? Wie kriege ich das bei Explain Everything rein?» (Frau T.; Grundschullehrerin in Gruppendiskussion)

Innerhalb unseres Samples stellt sich die Situation so dar, dass die meisten Lehrkräfte nach dem Einführungsworkshop zunächst versuchen, sich autodidaktisch in weitere Anwendungen des Tablets einzuarbeiten. Teilweise erfolgt dies, indem gezielt nach spezifischen Funktionen gesucht wird; teilweise werden aber im alltäglichen Umgang mit dem Gerät

5 Die daraus resultierende Macht des Herstellers und dessen weitreichender Einfluss auf die Institution Schule bedürfen sicherlich einer genaueren Reflexion, auf die im Rahmen dieses Beitrags aus Platzgründen lediglich verwiesen werden kann. 
auch ganz zufällig bislang unbekannte Funktionen entdeckt und für die den eigenen schulischen Gebrauch erschlossen. Mehrere Lehrkräfte verweisen darauf, im Rahmen ihrer autodidaktischen Bemühungen häufiger an Grenzen zu stossen und auf die spontane Unterstützung von Kollegen, technikkundigen Familienmitgliedern oder Freunden angewiesen zu sein. Insgesamt lässt sich festhalten, dass die tentativen Austauschpraktiken, die nach dem Einführungsworkshop stattfinden, so gut wie ausschliesslich von non-formalen und informellen Lern- bzw. Unterstützungsprozessen geprägt sind. Kritisch wird das Fehlen eines formalen Rahmens immer dann, wenn auftretende Probleme mit dem Gerät von einer Lehrkraft allein durch Ausprobieren nicht (oder allenfalls unter unverhältnismässigem Aufwand) gelöst werden können und dann aber die notwendige Hilfestellung aus dem Kollegenkreis oder dem privaten Umfeld ausbleibt. Ohne geeignete Unterstützung kann schlimmstenfalls das Zustandekommen des Hybrid-Akteurs aus Lehrkraft und Tablet gänzlich verhindert, zumindest aber die Ausbildung bestimmter Dingfunktionen für berufliche Kontexte (vgl. Punkt 2) blockiert werden. Unsere Datenanalyse zeigt, dass der einmalige Einführungsworkshop seitens der Lehrkräfte zwar als hilfreich, aber nicht ausreichend empfunden wird. Offensichtlich besteht erheblicher Bedarf an weiteren formalen Hilfsangeboten, die tabletbezogene Aneignungsprozesse systematisch dort unterstützen, wo tentative Praktiken ohne formale Begleitung an ihre Grenzen stossen:

«Ja, wenn er [gemeint ist der Medienbildungsreferent der Stadt, der auch die Fortbildungen durchführt] vielleicht noch ein paar Mal mehr gekommen wäre, so ne? Das war eine Einführung erstmal. [...] Aber wenn man dann eine Frage hat und man kennt sich damit gar nicht aus, ähm, dann muss man eben ausprobieren oder muss irgendwer [bei der zuständigen Stelle der Stadt] anrufen, kontaktieren, also das war [...] schwierig zum Teil.» (Frau D.; Grundschullehrerin/Medienbeauftragte im Einzelinterview)

Insgesamt ist festzuhalten, dass viele Lehrkräfte einen institutionalisierten, d. h. seitens der Schule bereitgestellten formalen Rahmen vermissen, der den erfolgreichen Verlauf der Tabletaneignung fortlaufend absichert. 
Vor diesem Hintergrund scheint es zunächst widersprüchlich, dass unsere Analysen andererseits aber eine geringe Bereitschaft zur Teilnahme an formalen Fortbildungsveranstaltungen erkennen lassen. Auffällig wenige Lehrerinnen und Lehrer unseres Samples berichten, zusätzlich zu dem verpflichtenden Einführungsworkshop noch weitere Fortbildungsveranstaltungen zu tabletbezogenen Themen besucht zu haben.

Eine erste mögliche Ursache der geringen Fortbildungsbeteiligung deutet sich im Interview mit einer Medienbeauftragten an:

«Wir haben eine Fortbildungsbeauftragte [...] mit der sprechen wir dann ab, Mensch, was sind für Fortbildungen sonst noch gewünscht [...] also sie macht Abfragen bei den Lehrern [...] wenn dann was kommt mit iPads, dann gucke ich mich um oder Herr H. je nachdem oder wir halt in der Gruppe gucken uns um ähm was wird angeboten, wo kann man sich melden.» (Frau D.; Grundschullehrerin/Medienbeauftragte im Einzelinterview)

Die hier beschriebene Vorgehensweise, bei den Lehrkräften Fortbildungsbedarfe zunächst abzufragen und dann lediglich zu explizit gewünschten Themen entsprechende Veranstaltungen anzubieten, erscheint insbesondere im Bereich der medienpädagogischen Lehrerinnen- und Lehrerfortbildung problematisch: Um die eigenen Bedarfe konkret benennen zu können, bedarf es gewisser Vorkenntnisse, die aber gerade in Bezug auf digitale Medien bei Lehrkräften noch nicht mehrheitlich vorausgesetzt werden können (vgl. Punkt 4.2.2).

In anderen Interview- und Gruppendiskussionspassagen tritt als eine zweite mögliche Ursache der geringen Fortbildungsbeteiligung die Zeitknappheit in den Mittelpunkt: An verschiedenen Stellen wird deutlich, dass das Thema Tableteinsatz in Konkurrenz zu zahlreichen anderen wichtigen Fortbildungsthemen steht. Möglicherweise führt dies dazu, dass Lehrkräfte selbst dann, wenn sie einen tabletbezogenen Fortbildungsbedarf klar formulieren könnten, bewusst davon absehen, weil sie sich zeitlich ohnehin schon zu stark eingebunden fühlen.

Eine dritte mögliche Ursache dafür, dass viele Lehrkräfte über den allgemeinen Einführungsworkshop hinaus kaum noch weiterführende Tablet-Fortbildungen besuchen, scheint darin zu liegen, dass sie keine 
passgenau zugeschnittenen Veranstaltungen finden. Dies wiederum ist vermutlich dadurch bedingt, dass tabletbezogene Fortbildungsinteressen je nach medienbezogenen Vorkenntnissen, nach Unterrichtsfach, nach Funktion innerhalb der Schule usw. so stark variieren, dass es schlicht unmöglich ist, jedem gerecht zu werden. Die folgenden Interviewpassagen spiegeln die grosse Heterogenität innerhalb der Kollegien wider:

«Datensicherheit, das wäre eine Fortbildung, auf jeden Fall. Das ist so ein grosses Thema.» (Frau D.; Grundschullehrerin/Medienbeauftragte im Einzelinterview)

«Aber wirklich unterrichtspraktisch [...] ich sag mal als Beispiel zweite Klasse, Thema Wiese und was könnte man da jetzt beispielsweise für zwei drei neuere Methoden mit dem iPad da tun.» (Frau B.; Grundschullehrerin im Einzelinterview)

«Also ich kann mir vorstellen, dass auch im kreativen Bereich ähm also hier schulintern mit den iPads ähm, indem ich hier Zeichenprogramme aufrufen kann, mit denen ich noch nicht gut arbeiten kann, wo ich erklärt bekommen möchte wie kann ich jetzt ein Kind im zweiten Schuljahr eigentlich hier ähm die Schreibschrift oder die Grundschrift vermitteln, mit dem iPad.» (Frau G.; Grundschullehrerin im Einzelinterview)

«Ich persönlich würde gerne mehr in diese, mehr über diese Apps erfahren, was du eben nutzt, B., ne? Diese Klassen-Apps und was du vorhin erklärt hast, ne? Diese verschiedenen Sachen, weil ich mich damit nicht auskenne.» (Herr J.; Grundschullehrer in Gruppendiskussion)

Die hier zum Ausdruck gebrachten individuellen Fortbildungsinteressen lassen sich nur schwer bündeln, sodass die Organisation gemeinsamer Schulungen für alle Lehrkräfte einer Schule schwer umsetzbar ist.

Zusammenfassend gesprochen scheint das Format der klassischen Fortbildungsveranstaltung also aus drei Gründen weniger gut geeignet zu sein, um tabletbezogenen Aneignungsprozessen den notwendigen 
formalen Rahmen zu geben: Erstens hindert unzureichendes Grundlagenwissen die Lehrkräfte an einer gezielten Suche nach sinnvollen weiterführenden Angeboten; zweitens mangelt es an zeitlichen Ressourcen; und drittens erschwert die hohe Diversität der Bedarfe innerhalb von Kollegien die Organisation passgenauer Angebote.

Wie unsere Datenauswertung zeigt, beginnen sich an den Schulen im Zuge der Tabletimplementation aber bereits alternative Formate formalen Lernens bzw. formaler Lernunterstützung zu etablieren: Eine der Schulen hat eine Art «Tabletsprechstunde» eingeführt, in der medienaffine Lehrkräfte ihren Kolleginnen und Kollegen regelmässig bei der Problembewältigung helfen. In einer anderen Schule wurde innerhalb von Konferenzen ein festes Zeitfenster eingerichtet, innerhalb dessen ausgewählte BestPractice-Beispiele präsentiert werden. Es handelt sich dabei um eine Art schulinterne Kurzfortbildung, bei der Lehrpersonen, die zuvor stellvertretend für den Rest des Kollegiums an einer externen Fortbildungsveranstaltung teilgenommen haben, ihre Kenntnisse gebündelt und bedarfsgerecht weitergeben. Diese Massnahmen werden von den Lehrkräften unseres Samples grundsätzlich positiv beurteilt, wobei jedoch mehrfach betont wird, dass es sich allenfalls um erste Lösungsansätze handele, die weiter optimiert und verstetigt werden sollten.

\section{Zwischenfazit und Ausblick}

Insgesamt erscheinen die in unseren Interview-, Gruppendiskussions- und Beobachtungsdaten deutlich werdenden Prozesse der Tabletaneignung bzw. auch die als besonders aneignungsrelevant identifizierten Einflussfaktoren wenig schulformspezifisch. Zwar beziehen sich alle uns bislang zur Verfügung stehenden Daten auf grundschultypische Lehr-/Lernsettings, was bedeutet, dass z. B. nur von bestimmten, im Grundschulbereich sinnvoll einsetzbaren Apps die Rede ist. Abgesehen davon ist aber anzunehmen, dass die prinzipiellen Abläufe und Zusammenhänge der Tabletaneignung von Lehrkräften, die unter Punkt 4 herausgearbeitet wurden, so oder in sehr ähnlicher Form auch bei entsprechenden Erhebungen im Sekundarschulbereich feststellbar sein dürften. ${ }^{6}$

6 Letzteres bleibt freilich im Rahmen von Anschlussstudien unter Einbeziehung weiterer Schulformen zu überprüfen. 
Abschliessend sollen auf Basis der bislang vorliegenden Analyseergebnisse erste Implikationen für die Förderung der Tabletaneignung von Lehrkräften gezogen werden. Sinnvoll erscheint es, bei den als besonders aneignungsrelevant identifizierten Einflussfaktoren anzusetzen, sodass nachfolgend Empfehlungen für die drei Bereiche Grundhaltung zu digitalen Medien im Schulkontext, Medienkompetenz und diesbezügliche Selbsteinschätzung sowie Unterstützung im Verlauf der Aneignung (vgl. Punkt 4.2) ausgesprochen werden. ${ }^{7}$

Was den ersten zentralen Einflussfaktor, die Grundhaltung zu digitalen Medien im Schulkontext, angeht, treffen wir bei den Lehrkräften unseres Samples bereits auf nahezu ideale Ausgangsbedingungen. Die seitens der Schulleitungsebene gezeigte Aufgeschlossenheit spiegelt sich bereits in den persönlichen Einstellungen der einzelnen Lehrkräfte wider, die dem Tableteinsatz zu Beginn allesamt offen gegenüberstehen. Wichtig scheint uns, dass die feineren Nuancen dieser grundsätzlich offenen Haltung stärker berücksichtigt werden. So bewegen sich die persönlichen Einstellungen unserer Untersuchungsteilnehmenden in einem Spektrum von freudig-erwartungsvoller bis hin zu kritischer Aufgeschlossenheit. Um etwaigen Enttäuschungen und einem daraus resultierenden Verlust der zunächst positiven Grundhaltung vorzubeugen, sollte den Lehrkräften in Vorgesprächen hinreichend Gelegenheit gegeben werden, eventuell überzogene Erwartungen oder auch Befürchtungen zu thematisieren und zu reflektieren. Eine von Anbeginn realistische Einschätzung von Chancen wie auch Risiken der Tabletnutzung kann erheblich dazu beitragen, die positive Grundhaltung gegenüber dem Gerät dauerhaft zu erhalten. Darüber hinaus scheint es geboten, im Rahmen solcher Vorgespräche über die persönlichen medienbezogenen Einstellungen der Lehrkräfte vor allem auch den Aspekt der Verantwortung gegenüber Kindern und Jugendlichen in

7 Es sei darauf hingewiesen, dass es sich um erste vorbehaltlich ausgesprochene Empfehlungen handelt. Sie wurden auf Basis von Forschungsdaten erarbeitet, deren Aussagekraft sich auf die Anfangsepisode der Tabletaneignung (d. h. primär auf die erste Ebene der Herausbildung des Hybrid-Akteurs aus Lehrkraft und Tablet, siehe Abb.1, 1. Ebene) beschränkt. Inwieweit diese Empfehlungen darüber hinaus auch für den weiteren Verlauf der Tabletaneignung erfolgsförderlich sein können, müssen anschliessende Forschungsarbeiten (unter Einbezug der Daten des geplanten zweiten Erhebungszeitraums) zeigen. 
Zeiten einer tiefgreifenden Mediatisierung bewusst(er) zu machen, um mediale Aneignungsprozesse primär von dieser Seite her zu motivieren (und nicht allein durch Aspekte wie Arbeitserleichterung, Zeiteinsparung etc.).

Unsere Empfehlungen in Bezug auf den zweiten wesentlichen Einflussfaktor, die allgemeine Medienkompetenz und die diesbezügliche Selbsteinschätzung der Lehrkraft, zielen darauf, schnell und anhaltend Erfolgserlebnisse im Umgang mit dem Tablet zu gewährleisten. Dies setzt zweierlei notwendig voraus: Zum einen sollte bei jeder Lehrkraft zunächst sichergestellt werden, dass medienbezogene Basiskompetenzen auf einem gewissen Level vorhanden sind, bevor man das Tablet zur selbstständigen Nutzung aushändigt. Je nach Ausgangslevel reicht dafür ein einmaliger Einführungsworkshop womöglich nicht aus, d. h. es bedarf hier eines differenzierteren Angebots als bisher. Zum anderen gilt es, medienbezogene Stereotypen gezielt anzusprechen und argumentativ zu entkräften (wie z. B. besagte Generationszuschreibungen, siehe Punkt 4.2.2). Es ist die Kombination aus beidem - einer vorsorglichen Vermittlung hinreichender Basiskompetenzen und einer Hinführung zur reflektierten Selbsteinschätzung - die dazu führt, dass Effekte wechselseitig positiver Verstärkung zwischen Medienkompetenz und diesbezüglichem Selbstbewusstsein entstehen können, womit ideale Bedingungen für den gesamten weiteren Verlauf des Aneignungsprozesses geschaffen wären.

Hinsichtlich des dritten wichtigen Einflussfaktors, der Unterstützung der Lehrkraft im Verlauf der Aneignung, empfehlen wir, sowohl den Bereich des non-formalen und informellen tabletbezogenen Lernens als auch den Bereich des formalen tabletbezogenen Lernens weiter zu verbessern, weil eine Mixtur der verschiedenen Lernformen dem aktuellen Stand der Forschung zufolge mit den besten Erfolgsaussichten verbunden ist (siehe Punkt 4.2.3).

Bezüglich non-formaler und informeller Lernvorgänge konnten wir feststellen, dass viele Lehrkräfte während ihrer autodidaktischen Auseinandersetzung mit dem Tablet stellenweise überfordert sind und sich dann selbst Hilfe organisieren müssen, um allein nicht lösbare Probleme zu überwinden und im Aneignungsprozess voran zu kommen. Bislang wenden sich die Lehrkräfte nach eigener Aussage in solchen Situationen entweder an technikaffine Kolleginnen und Kollegen oder an Kundige aus 
dem privaten Umfeld. So entsteht ein hohes Mass an Abhängigkeit von Dritten. Eine sinnvolle Massnahme könnte darin bestehen, die Lehrkräfte stärker zur Selbsthilfe unter Nutzung frei verfügbarer Online-Ressourcen zu befähigen. Interessanterweise wird das an allen Schulen unseres Samples flächendeckend verfügbare Internet, das wie kein anderes Medium mit tiefgreifender Mediatisierung assoziiert ist (vgl. Hepp 2016), an keiner Stelle des bislang erhobenen Datenmaterials als potentielle Ressource zur situativen Problemlösung oder als Inspirationsquelle für didaktisch sinnvolle Einsatzmöglichkeiten benannt. Hilfreich wäre womöglich schon, systematisch auf geeignete Info-Seiten, Erklärvideos etc. hinzuweisen (z. B. in Form eines regelmässig herausgegebenen Newsletters).

Formales Lernen, so eine weitere Erkenntnis unserer Analysen, kommt im Verlauf der Tabletaneignung bislang zu kurz. Das Tablet stellt die einzelne Lehrkraft durch seine permanente Weiterentwicklung im Zusammenhang mit der hohen technischen Innovationsdichte auf Softwareebene stetig vor neue Handlungsprobleme, die fortlaufend einer zielgerichteten Begleitung bedürfen. Die Lehrkräfte unseres Samples weisen selbst explizit darauf hin, dass ihren tabletbezogenen Aneignungsprozessen ein über den Einführungsworkshop hinausreichender formaler Rahmen fehlt, der jedoch unabdingbar ist, um non-formale und informelle Lernvorgänge systematisch zu ergänzen und den Erfolg der Tabletaneignung institutionsseitig abzusichern. Formales Lernen bzw. formale Lernunterstützung in Form von klassischen Fortbildungsformaten scheint allerdings speziell im Bereich Tabletaneignung aus mehreren Gründen wenig praktikabel (vgl. Punkt 4.2.3). Stattdessen scheinen sich verschiedene innovative Formen von 〈Kurzfortbildungen〉 durchzusetzen, die formale Lerngelegenheiten bieten, dabei aber im Vergleich zur klassischen Fortbildung weniger aufwändig zu organisieren sind, zeitlich kürzer gehalten werden können und sich inhaltlich flexibler auf die heterogenen Bedarfe innerhalb der Kollegien zuschneiden lassen. Vor diesem Hintergrund lautet unsere Empfehlung, vielversprechende Ansätze dieser Art, wie z. B. die Tablet-Sprechstunde und die tabletbezogenen Best Practice-Slots in Konferenzen, zu verstetigen und bei Bedarf auszubauen. Ausserdem empfehlen wir eine inhaltliche Optimierung dahingehend, dass neben rein technischen Aspekten verstärkt didaktische (und bestenfalls auch spezifisch fachdidaktische) Aspekte 
gemeinsam bearbeitet werden sollten, um sich gegenseitig zu neuen Nutzungsweisen zu inspirieren und diesbezüglich die Entstehung routinierter Austauschpraktiken zwischen Lehrkraft und Tablet gezielter zu fördern.

Des Weiteren ist auch zu Zwecken formalen Lernens bzw. formaler Lernunterstützung unbedingt zu empfehlen, den Lehrkräften unterstützende Online-Angebote zu unterbreiten. Überraschenderweise scheint diese Option seitens der Schulen unseres Samples - trotz einer hohen Aufgeschlossenheit der Schulleitungen für Digitalisierungsinitiativen - bislang noch gar nicht genutzt zu werden. Dabei liessen sich gerade auch digital innovative formale Fortbildungsmöglichkeiten schaffen, die im Vergleich zu klassischen Präsenzfortbildungen organisatorisch, zeitlich und inhaltlich flexibler gestaltet werden könnten und somit den Wünschen der Lehrkräfte entgegenkämen. Sinnvoll könnte zum Beispiel sein, E-Learning-Materialien in Form von digitalen Checklisten, Leitfäden, Video-Tutorials u.. ä. für Selbstlernphasen der Lehrkräfte bereitzustellen. Wichtig wäre, zugleich immer auch Kommunikationstools für den Austausch aller Beteiligten einzubinden. Auch Beratungsangebote wie die Tablet-Sprechstunde könnten zumindest teilweise digitalisiert werden. Um zeitlich und räumlich flexibler arbeiten zu können, wäre es denkbar, mittels eines entsprechenden Tools den Bildschirm zu teilen und so bestimmte Anwendungen gemeinsam zu simulieren. Auch bei Online-Angeboten sollte grundsätzlich berücksichtigt werden, dass neben den Anforderungen auf technischer Ebene die (fach-)didaktischen Aspekte der Tabletnutzung nicht zu kurz kommen.

Zusammenfassend bleibt festhalten, dass alle erarbeiteten Vorschläge zur Optimierung non-formaler und informeller wie auch formaler Lernvorgänge im Laufe der Tabletaneignung auf eine noch effektivere Unterstützung von Lehrkräften zielen. Erfolgsentscheidend ist, dass die Lehrkräfte, wann immer nötig, bedarfsgerechte Impulse zur Auseinandersetzung mit den jeweils neuesten technisch möglichen und pädagogisch sinnvollen Anwendungen des Tablets im Arbeitsalltag bekommen. 


\section{Literatur}

Allert, Heidrun, und Michael Asmussen. 2017. "Bildung als produktive Verwicklung». In Digitalität und Selbst: Interdisziplinäre Perspektiven auf Subjektivierungs- und Bildungsprozesse, herausgegeben von Heidrun Allert, Michael Asmussen, und Christoph Richter, 27-68. Bielefeld: Transcript. https://doi. org/10.14361/9783839439456-004.

Bastian, Jasmin, und Stefan Aufenanger, Hrsg. 2017. Tablets in Schule und Unterricht. Forschungsmethoden und -perspektiven zum Einsatz digitaler Medien. Wiesbaden: Springer VS. https://doi.org/10.1007/978-3-658-13809-7.

Burgfeld-Meise, Bianca, und Dorothee M. Meister. 2020. "Generationsspezifische Medienzugänge. Medien - Biographie - Generation». In Jugend, Familie und Generationen im Wandel. Erziehungswissenschaftliche Facetten, hrsg. v. Thosten Fuchs, Anja Schierbaum, und Alena Berg, 329-343. Wiesbaden: Springer VS. https://doi.org/10.1007/978-3-658-24185-8_20.

Couldry, Nick. 2008. «Actor Network Theory and media. Do they connect and on what terms?». In Connectivity, networks and flows. Conceptualizing contemporary communications, herausgegeben von Andreas Hepp, Friedrich Krotz, Shaun Moores, und Carsten Winter, 93-110, Cresskill: Hampton Press.

Dehmel, Lukas, und Sebastian Zick. 2020. «Das Smartphone in der berufsvorbereitenden Bildung junger Erwachsener - Empirische Erkundungen aus praxistheoretischer Perspektive». In Praxistheoretische Perspektiven in der Medienpädagogik, herausgegeben von Patrick Bettinger und Kai-Uwe Hugger, 6:179-196. Digitale Kultur und Kommunikation. Wiesbaden: Springer Fachmedien Wiesbaden. https://doi.org/10.1007/978-3-658-28171-7_10.

Dewey, John. 1989. «Peirce's theory of linguistic signs, thought, and meaning». In John Dewey. The Later Works 1925-1953, herausgegeben von Jo Ann Boydston, 141-152. Carbondale: Southern Illinois University Press.

Drossel, Kerstin, Birgit Eickelmann, Heike Schaumburg, und Amelie Labusch. 2019. «Nutzung digitaler Medien und Prädiktoren aus der Perspektive der Lehrerinnen und Lehrer im internationalen Vergleich». In ICILS 2018 \#Deutschland. Computer- und informationsbezogene Kompetenzen von Schülerinnen und Schülern im zweiten internationalen Vergleich und Kompetenzen im Bereich Computational Thinking, herausgegeben von Birgit Eickelmann, Wilfried Bos, Julia Gerick, Frank Goldhammer, Heike Schaumburg, Knut Schwippert, Martin Senkbeil, Jan Vahrenhold, 205-240. Münster: Waxmann.

Eickelmann, Birgit, Wilfried Bos, und Amelie Labusch. 2019. «Die Studie ICILS 2018 im Überblick. Zentrale Ergebnisse und mögliche Entwicklungsperspektiven». In ICILS 2018 \#Deutschland. Computer- und informationsbezogene Kompetenzen von Schülerinnen und Schülern im zweiten internationalen Vergleich und Kompetenzen im Bereich Computational Thinking, herausgegeben von Birgit Eickelmann, Wilfried Bos, Julia Gerick, Frank Goldhammer, Heike Schaumburg, Knut Schwippert, Martin Senkbeil, Jan Vahrenhold, 7-32. Münster: Waxmann. 
Friedrich, Katja, Friederike Siller, und Albert Treber, Hrsg. 2015. Smart und mobil. Digitale Kommunikation als Herausforderung für Bildung Pädagogik und Politik. München: Kopaed.

Hartmann, Maren, und Jeffrey Wimmer. 2011. «Digitale Medientechnologien. Vergangenheit - Gegenwart - Zukunft». In Digitale Medientechnologien. Vergangenheit - Gegenwart - Zukunft, herausgegeben von Maren Hartmann, und Jeffrey Wimmer, 7-26, Wiesbaden: VS Verlag. https://doi.org/10.1007/978-3531-93275-O_1.

Hepp, Andreas. 2016. "Kommunikations- und Medienwissenschaft in datengetriebenen Zeiten». Publizistik 61 (3): 225-246. https://doi.org/10.1007/s11616016-0263-y.

Hepp, Andreas, und Uwe Hasebrink. 2017. «Kommunikative Figurationen. Ein konzeptioneller Rahmen zur Erforschung kommunikativer Konstruktionsprozesse in Zeiten tiefgreifender Mediatisierung». Medien \& Kommunikationswissenschaft 65 (2), 330-347. https://doi.org/10.5771/1615-634X-2017-2-3030.

Hepp, Andreas, und Uwe Hasebrink. 2018. «Researching Transforming Communications in Times of Deep Mediatization. A Figurational Approach». In Communicative Figurations. Transforming Communications in Times of Deep Mediatization, herausgegeben von Andreas Hepp, Andreas Breiter, und Uwe Hasebrink, 15-50. Basingstoke: Palgrave Macmillan. https://doi.org/10.1007/9783-319-65584-0_2.

Hüther, Jürgen. 2005. «Neue Medien». In Grundbegriffe Medienpädagogik. 4. Auflage, herausgegeben von Jürgen Hüther, und Bernd Schorb, 345-351, München: Kopaed.

Ifenthaler, Dirk. 2018. «How we learn at the digital Workplace». In Digital Workplace Learning. Bridging formal and informal Learning with digital Technologies, herausgegeben von Dirk Ifenthaler, 3-8. Cham: Springer Nature.

Klein, Anika. 2017. «Der Weg ins digitale Netz - eine Frage des Alters und der Generation? Ergebnisse einer qualitativen Studie zur Bedeutung von Alters- und Generationsstereotypen für den Umgang mit digitalen Medien». In Jahrbuch Medienpädagogik 13. Vernetzt und entgrenzt. Gestaltung von Lernumgebungen mit digitalen Medien, herausgegeben von Kerstin Mayrberger, Johannes Fromme, Petra Grell, und Theo Hug, 61-77. Wiesbaden: Springer VS. https://doi. org/10.1007/978-3-658-16432-4_5.

KMK. 2016. «Bildung in der digitalen Welt. Strategie der Kultusministerkonferenz». https://www.kmk.org/fileadmin/Dateien/pdf/PresseUndAktuelles/2017/Strategie_neu_2017_datum_1.pdf.

Kommer, Sven. 2016. "Buch statt Tablet-PC. Warum die digitalen Medien nicht in die Schule kommen - der Faktor LehrerIn». In Wi(e)derstände. Digitaler Wandel in Bildungseinrichtungen, herausgegeben von Thomas Knaus, und Olga Engel, 35-67. München Kopaed.

Kommission der Europäischen Gemeinschaften. 2000. Memorandum über Lebenslanges Lernen. Brüssel. https://www.die-bonn.de/esprid/dokumente/doc2000/EUOO_01.pdf. 
Krotz, Friedrich. 2001. Die Mediatisierung des kommunikativen Handelns. Der Wandel von Alltag und sozialen Beziehungen, Kultur und Gesellschaft durch die Medien. Wiesbaden: Westdeutscher Verlag. https://doi.org/10.1007/978-3-322-90411-9.

Krotz, Friedrich. 2017. «Mediatisierung. Ein Forschungskonzept». In Mediatisierung als Metaprozess. Transformationen, Formen der Entwicklung und die Generierung von Neuem, herausgegeben von Friedrich Krotz, Cathrin Despotović und Merle-Marie Kruse, 13-32. Wiesbaden: Springer VS. https:// doi.org/10.1007/978-3-658-16084-5_2.

Kuckartz, Udo. 2016. Qualitative Inhaltsanalyse. Methoden, Praxis, Computeruntersützung. 3. Auflage. Weinheim/Basel: Beltz Juventa.

Latour, Bruno. 1998. Wir sind nie modern gewesen. Versuch einer symmetrischen Anthropologie. Frankfurt a.M.: Suhrkamp.

Meister, Dorothee M., und Ilka Mindt, Hrsg. 2020. Mobile Medien im Schulkontext. Bd. 41. Medienbildung und Gesellschaft. Wiesbaden: Springer Fachmedien Wiesbaden. https://doi.org/10.1007/978-3-658-29039-9.

Nohl, Arnd-Michael. 2011. Pädagogik der Dinge. Bad Heilbrunn: Klinkhardt.

Nohl, Arnd-Michael. 2013. "Sozialisation in konjunktiven, organisierten und institutionalisierten Transaktionsräumen. Zum Aufwachsen mit materiellen Artefakten». Zeitschrift für Erziehungswissenschaft 16 (2), 189-202. https://doi. org/10.1007/s11618-013-0419-8.

Nohl, Arnd-Michael, und Christoph Wulf. 2013. «Die Materialität pädagogischer Prozesse zwischen Mensch und Ding». Zeitschrift für Erziehungswissenschaft 16 (2), 1-13. https://doi.org/10.1007/s11618-013-0406-0.

Petko, Dominik. 2012. "Hemmende und förderliche Faktoren des Einsatzes digitaler Medien im Unterricht. Empirische Befunde und forschungsmethodische Probleme». In Jahrbuch Medienpädagogik 9, herausgegeben von Renate SchulzZander, Birgit Eickelmann, Heinz Moser, Horst Niesyto, und Petra Grell, 2950, Wiesbaden: Springer VS. https://doi.org/10.1007/978-3-531-94219-3_3.

Rammert, Werner, und Ingo Schulz-Schaeffer. 2002. "Technik und Handeln. Wenn soziales Handeln sich auf menschliches Verhalten und technische Abläufe aufteilt». In Können Maschinen handeln? Soziologische Beiträge zum Verhältnis von Mensch und Technik, herausgegeben von Werner Rammert, und Ingo Schulz-Schaeffer, 11-64. Frankfurt a.M. Campus.

Tillmann, Alexander. 2018. «Begleituntersuchung zum Projekt MOLE. Mobiles Lernen in Hessen». In Tablet-Klassen. Begleituntersuchung, Unterrichtskonzepte und Erfahrungen aus dem Pilotprojekt 'Mobiles Lernen in Hessen' - MOLE», herausgegeben von Alexander Tillmann, und Ingo Antony, 13-40, Münster: Waxmann.

Welling, Stefan. 2017. «Methods matter. Methodisch-methodologische Perspektiven für die Forschung zum Lernen und Lehren mit Tablets». In Tablets in Schule und Unterricht. Forschungsmethoden und -perspektiven zum Einsatz digitaler Medien, herausgegeben von Jasmin Bastian, und Stefan Aufenanger, 15-36, Wiesbaden: Springer VS. https://doi.org/10.1007/978-3-658-13809-7_2. 